\title{
TREINAMENTO EM CIRURGIA DE CATARATA EM SIMULADORES DE REALIDADE VIRTUAL
}

\section{ARTIGO DE REVISÃO}

SAVIAN, Tiago Rezende ${ }^{1}$

FRAGA, Carolina Cândida De Resende ${ }^{2}$

FRAGA, Ana Laísa Cândida De Resende ${ }^{3}$

SAVIAN, Tiago Rezende. FRAGA, Carolina Cândida De Resende. FRAGA, Ana Laísa Cândida De Resende. Treinamento em cirurgia de catarata em simuladores de realidade virtual. Revista Científica Multidisciplinar Núcleo do Conhecimento. Ano 05, Ed. 01, Vol. 06, pp. 05-22. Janeiro de 2020. ISSN: 2448-0959, Link de acesso: https://www.nucleodoconhecimento.com.br/saude/treinamento-em-cirurgia

\section{RESUMO}

Os programas de treinamento de oftalmologia responderam rapidamente à necessidade de avaliação e aprimoramento desenvolvendo uma variedade de ferramentas de avaliação e de aperfeiçoamento cirúrgico. A cirurgia de catarata com

\footnotetext{
${ }^{1}$ Médico Generalista graduado pela UNIMES - Universidade Metropolitana de Santos, cidade de Santos/SP. Advogado e bacharel em direito graduado pelo CESUR - Centro de Ensino Superior de Rondonópolis, cidade de Rondonópolis/MT. Pós-graduado em Direito Processual do Trabalho pela UGF - Universidade Gama Filho, Cidade do Rio de Janeiro/RJ.

2 Fisioterapeuta graduada pela PUC-GO - Pontifícia Universidade Católica de Goiás, cidade de Goiânia/GO. Acadêmica do $6^{\circ}$ ano do curso de medicina da UNIMES Universidade Metropolitana de Santos, cidade de Santos/SP.

${ }^{3}$ Médica Veterinária graduada e Mestre pela UFMT - Universidade Federal do Mato Grosso, cidade de Cuiabá/MT. Acadêmica do $3^{\circ}$ ano do curso de medicina da FAMP - Faculdade Morgana Potrich, cidade de Mineiros/GO.
} 
facoemulsificação é um procedimento desafiador para cirurgiões que estão em treinamento para aprimoramento de segurança, eficiência e precisão. No presente estudo foram discutidas as ferramentas de aprendizado auxiliares, por diversos autores, fora e na sala de cirurgia que os programas de residência e de estudo e aprimoramento continuado incorporaram em seu currículo para melhorar as habilidades cirúrgicas. Foram escolhidos estudos publicados entre janeiro de $2014 \mathrm{e}$ junho de 2019 que estavam disponíveis no idioma inglês, em texto completo na base de dados eletrônica Medline/Pubmed. A aplicação de simuladores de RV não é uma tecnologia totalmente nova, mas sua aplicação na área de cirurgia de catarata por facoemulsificação para fins de treinamento e avaliação cirúrgicos virtuais ainda está em sua fase inicial. Os resultados dos estudos experimentais apresentados nesta revisão de literatura demonstram que os simuladores de RV têm o potencial e a capacidade de serem aplicados como uma ferramenta viável de avaliação de proficiência nos quatro principais procedimentos completos da cirurgia de catarata por facoemulsificação.

Palavras-chave: Procedimentos cirúrgicos oftalmológicos, catarata, realidade virtual, facoemulsificação, simulação computacional.

\section{INTRODUÇÃO}

Em um esforço para afastar as dificuldades associadas a avaliações qualitativas, subjetivas e de preceptores atrasados, as ferramentas de realidade virtual (RV) foram projetadas para avaliar a habilidade cirúrgica de estudantes, residentes e médicos de maneira oportuna e objetiva.

As avaliações aparecem na forma de resultados peri e pós-operatórios, ajustes, laboratórios, avaliações em vídeo, registros de procedimentos auto relatados, simuladores de realidade virtual, sistemas de análise de movimento, listas de verificação específicas de procedimentos e escalas globais de classificação, no presente estudo foram avaliados os simuladores de realidade virtual. 
Dentre os simuladores de realidade virtual, três dispositivos de simulação foram desenvolvidos para uso em cirurgia de catarata: Eyesi® (VRmagic, da Alemanha), PhacoVision® (Melerit Medical, da Suécia) e MicrovisTouch ${ }^{\circledR}$ (ImmersiveTouch, dos EUA). A maioria dos estudos publicados na literatura utiliza o simulador Eyesi®.

Foi relatado[4] que esse dispositivo fornece treinamento cirúrgico sistemático, eficaz e confiável com custo mais baixo. Há poucos estudos sobre os simuladores MicrovisTouch $\AA$ e PhacoVision $\AA$. As características do MicrovisTouch ${ }^{\circledR}$ são as vantagens de receber feedback tátil e ter uma cabeça virtual ajustável, no entanto, este dispositivo possui apenas um estágio da capsulorrexe e nenhum dos outros módulos disponíveis no simulador Eyesi $\AA^{\circledR}$. O simulador de cirurgia de catarata (Eyesi®) é usado regularmente em treinamento cirúrgico para facilitar a transição para a aplicação prática.

A catarata é a principal causa de cegueira e a taxa cirúrgica de catarata é usada como um indicador indireto do acesso a serviços de catarata em um país. De acordo com dados expostos pela OMS estimou-se que 285 milhões de pessoas em todo o mundo sofrem algum tipo de deficiência visual, muitas (80\%) das quais são evitáveis[5].

Uma vez que muitos desses pacientes não recebem cuidados oftalmológicos adequados, são necessários estudos para investigar o ou os motivos existentes que sustentem essa barreira à esses cuidados. Em regiões da América Latina a falta de conscientização e os custos incontroláveis parecem ser as barreiras centrais para o atendimento ${ }^{2}$.

Em 2014, o Plano de Ação para a Prevenção da Cegueira e Deficiência Visual da Organização Pan-americana da Saúde (PAHO)[6], utilizou os dados de 'cobertura cirúrgica da catarata' (de estudos epidemiológicos sobre cegueira e deficiência visual na América Latina) como um indicador de acesso a serviços oftalmológicos.

Resultados de nove pesquisas diferentes em toda a América Latina mostraram que a cobertura de cirurgias de catarata foi menor em áreas rurais e com deficiências socioeconômicas, indicando iniquidades na distribuição de serviços oftalmológicos. 
Além disso, usando pesquisas transversais de saúde ocular, uma avaliação comparativa conduzida em sete países latino-americanos demonstrou que a prevalência de cegueira e deficiência visual moderada estavam concentradas nas áreas mais desfavorecidas socialmente, enquanto a cobertura cirúrgica de catarata e resultados ótimos da cirurgia de catarata estavam concentrados entre as áreas mais ricas e socialmente favorecidas[7].

E o que está causando esta desigualdade nos serviços? Segundo Wang et al.[8] a taxa cirúrgica da catarata e os indicadores econômicos estão intimamente associados, indicando a forte influência da disponibilidade de recursos na prestação de cuidados de saúde. Considerando esse relacionamento, é importante ser inovador na prestação de serviços de baixo custo e investir estrategicamente no desenvolvimento de capacidade para atender às necessidades cirúrgicas de catarata em ambientes com poucos recursos.

Portanto, a presente pesquisa fez-se importante ao apresentar as novas tecnologias em treinamento de estudantes de oftalmologia. Tendo como objetivo mostrar os estudos relacionados e os resultados deles em discussão.

\section{METODOLOGIA}

\subsection{ESTRATÉGIA DE PESQUISA}

Foram escolhidos estudos publicados entre janeiro de 2014 e junho de 2019 que estavam disponíveis no idioma inglês, em texto completo na base de dados eletrônica Medline/Pubmed. O limite em 2019 para inclusão de publicações foi para enfatizar novos estudos e propostas. A pesquisa incluiu termos do MeSH: Ophthalmology AND Ophthalmologic Surgical Procedures AND Cataract AND Virtual Reality AND Phacoemulsification AND computer simulation. 


\subsection{CRITÉRIOS DE ELEGIBILIDADE}

Estudos que abordavam o tema de forma primária. Foram considerados elegíveis para inclusão se envolveram os resultados de treinamento de residentes ou médicos em simuladores na cirurgia de catarata.

Aplicamos os seguintes critérios de exclusão: artigos anteriores à data mínima de inclusão, e que não tinham texto na íntegra para leitura. Foi dada preferência pelas publicações dos últimos 5 anos (2014 - 2019).

\subsection{SELEÇÃO DE ARTIGOS}

Os autores revisaram e selecionaram de forma independente, os títulos e resumos dos artigos e manuscritos para identificar estudos potencialmente relevantes que abordassem o tema. Pesquisas manuais não foram realizadas. Finalmente, cópias em texto integral dos estudos que tiveram atendido os resultados, descritores e objetivo de treinamento por realidade virtual foram consideradas.

\section{RESULTADOS}

No Pubmed Central foram elencados 13 estudos, onde foram lidos os resumos, e destes, 09 foram separados para a leitura na íntegra. Ainda foram incluídos 3 estudos que foram encontrados por referências e citação, totalizando 12 artigos. Como seguem abaixo:

Tabela 1 - Autores e obras elencadas para discussão

\begin{tabular}{|l|l|}
\hline Autor / Revista / Data da publicação & Título do artigo \\
\hline BERGQVIST et al. Acta ophthalmologica, v. & $\begin{array}{l}\text { Establishment of a validated } \\
\text { 92, n. 7, p. 629-634, 2014. }\end{array}$ \\
$\begin{array}{ll}\text { training programme on the Eyesi } \\
\text { cataract simulator. A } \\
\text { prospective randomized study. }\end{array}$ \\
\hline
\end{tabular}


CHUNG et al. Journal of Cataract \& Effect of fine-motor-skill Refractive Surgery, v. 43, n. 7, p. 915-922, activities on surgical simulator 2017. performance.

LAM; SUNDARAJ; SULAIMAN. Medicina A systematic review of (Kaunas, Lithuania), v. 49, n. 1, p. 1-8, phacoemulsification cataract 2013. surgery in virtual reality simulators.

LANDIS et al. Investigative Ophthalmology Impact of Surgical Simulator \& Visual Science, v. 56, n. 7, p. 130-130, Training on Patients' 2015.

Perceptions of Resident Involvement in Cataract Surgery.

NG et al. Clinical Ophthalmology (Auckland, Impact of virtual reality NZ), v. 12, p. 885, 2018. simulation on learning barriers of phacoemulsification perceived by residents.

OFLAZ; KÖKTEKIR; OKUDAN, Turkish Does Cataract Surgery journal of ophthalmology, v. 48, n. 3, p. 122, Simulation Correlate with Real2018. life Experience?

SADIDEEN et al. International Journal of Surgical experts: born or made? Surgery v. 11, n. 9, p. 773-778, 2013.

SIKDER et al. British Journal of Surgical simulators in cataract Ophthalmology, v. 98, n. 2, p. 154-158, surgery training. 2014.

STAROPOLI et al. Simulation in Surgical simulation training Healthcare, v. 13, n. 1, p. 11-15, $2018 . \quad$ reduces intraoperative cataract surgery complications among residents.

TECLE et al. World Neurosurgery. 2018 Enhancing Microsurgical Skills Through Deliberate Practice 
THOMSEN et al. Ophthalmology, v. 124, n. Operating room performance 4, p. 524-531, 2017. improves after proficiencybased virtual reality cataract surgery training.

WISSE et al. BMJ Simulation and The Eyesi simulator in training Technology Enhanced Learning, v. 3, n. 3, ophthalmology residents: results p. 111-115, 2017. of a pilot study on self-efficacy, motivation and performance.

Fonte: Autores (2019).

\section{DISCUSSÃO}

Sadideen et al.[9] abordaram em sua pesquisa se a expertise, ou perícia, seria inerente em determinados indivíduos para a cirurgia, sem o aprimoramento através de treinamento. $O$ conceito de perícia cirúrgica e os processos envolvidos em seu desenvolvimento são tópicos, e há um esforço constante para identificar medidas confiáveis de desempenho especializado em cirurgia. O estudo explorou se especialistas em cirurgia são "nascidos" ou "feitos", com referência à teoria educacional e literatura pertinente.

Concluem em primeiro plano que o talento inato desempenha um papel importante, mas é insuficiente por si só para produzir um especialista em cirurgia. Múltiplas teorias que exploram a aquisição de habilidades motoras e a memória são relevantes, e a teoria de Ericsson sobre o desenvolvimento da competência seguida pela auto-prática deliberada tem sido especialmente influente ${ }^{6}$.

Reiteram que habilidades psicomotoras e não técnicas são necessárias para a progressão considerando os currículos de treinamento atuais; os especialistas em cirurgia são especialistas adaptativos que se destacam neles. Cita que a literatura sugere que a perícia cirúrgica é alcançada através da prática; ou seja, especialistas cirúrgicos são feitos, não nascidos ${ }^{6}$. 
Reconhecem que uma compreensão mais profunda da natureza do desempenho de especialistas e seu desenvolvimento garantirá que os programas de treinamento em educação cirúrgica sejam da mais alta qualidade possível, e que os educadores cirúrgicos devem procurar desenvolver uma abordagem baseada em expertise, com desempenho de especialistas como referência ${ }^{6}$.

Em outro momento Sadideen et al. ${ }^{6}$ perguntam se os cirurgiões (os bons) podem ser "feitos" e argumenta que muito antes do conhecimento real se desenvolver, é preciso alcançar competência. Alguns podem naturalmente alcançar isso devido ao talento inato, ainda requerendo prática adequada, e de outra forma podem requerer muito mais prática para atingir o mesmo nível de competência. Mas e se a prática simples não resultar na obtenção de tal competência?

Os autores citam alguns estudos, onde se pode argumentar que estes são limitados pelo tipo e duração do treinamento que foi fornecido e só pode ser considerado como uma visão "instantânea" de um ambiente muito complexo e multifatorial. No entanto, confirmam que a existência de diferentes curvas de aprendizado entre os indivíduos é um reflexo de suas habilidades técnicas inatas. O principal desafio para o educador cirúrgico moderno é elaborar programas de treinamento que possam lidar com essas deficiências para cada indivíduo - independentemente de sua habilidade inata ${ }^{6}$.

Seguindo a linha de estudo das habilidades, Tecle et al.[10] investigaram a melhora de habilidades microcirúrgicas por meio das práticas deliberadas. As habilidades microcirúrgicas são extremamente importantes para o campo da neurocirurgia e outras especialidades cirúrgicas, como cirurgia plástica e cirurgia cardiotorácica. No entanto, o aprendizado de habilidades microcirúrgicas tornou-se cada vez mais desafiador devido à limitação de deveres residentes.

As habilidades microcirúrgicas são extremamente importantes para o campo da neurocirurgia e outras especialidades cirúrgicas, como cirurgia plástica e cirurgia cardiotorácica. No entanto, o aprendizado de habilidades microcirúrgicas tornou-se cada vez mais desafiador por causa das horas de serviço limitadas; descentralização do cuidado; e a proliferação de terapias alternativas (embora estas muitas vezes 
complementares). Então, os autores colocam que, aprofundar o potencial da prática deliberada pode influenciar o treinamento microcirúrgico ${ }^{7}$.

Bergqvist et al.[11] estabeleceram e avaliaram um programa de treinamento sistemático para ser incluído no currículo oftalmológico. Para isso, utilizaram estudantes de medicina $(n=20)$ faltando cerca de um ano para a graduação e sem experiência oftalmológica prévia neste estudo prospectivo e randomizados em dois grupos.

O grupo $A(n=10)$ completou o programa de treinamento do simulador de catarata Eyesi uma vez por semana durante 4 semanas, enquanto o Grupo B $(n=10)$ completou uma vez por semana na primeira e na última semana. Dois cirurgiões de catarata foram usados para determinar dois níveis diferentes de escores de referência. Escore por módulo analisado [dois níveis diferentes de Capsulorrexe (A e B), manobra hidráulica, facoemulsificação, dividir e conquistar, pontuação geral, tempo total, lesão da córnea, ruptura da cápsula e lesão da cápsula por ultrassom foram registrados ${ }^{8}$.

Como resultado, o Grupo A superou o Grupo B em vários módulos, alcançou um número significativamente maior de escores de referência $(p<0,01)$ e causou menos complicações com relação à ruptura da Cápsula $(p=0,01)$ e dano da Cápsula pela ultrassonografia $(p<0,05)$. Ambos os Grupos A e B melhoraram seu desempenho e também se tornaram mais eficientes ( $p<0,01$ para ambos os grupos). $O$ grupo $A$ apresentou curva de aprendizado positiva para escore total ( $p<0,01)$, capsulorrexe $A$ $(p<0,01)$, capsulorrexe $B(p<0,01)$ e manobra hidráulica $(p=0,01)$. $O$ grupo $B$ apresentou melhora significativa para o escore geral $(p<0,01)$, manobra e $o$ facoemulsificação $(p=0,02)$ dividiram e apresentaram o escore $(p<0,01)$. Com os resultados os autores concluíram que o treinamento repetitivo com um programa de treinamento sistemático, baseado em módulos validados no simulador Eyesi, mostrou melhorar as habilidades simuladas na cirurgia de catarata. Um maior nível de habilidades e mais pontos de referência foram alcançados com mais treinamento. Além disso, o programa foi otimizado para ser aplicado no currículo oftalmológico padrão para treinamento em cirurgia de catarata ${ }^{8}$. 
$\mathrm{Ng}$ et al.[12] fizeram um curso piloto de simulação de cirurgia de catarata em ambiente virtual em Hong Kong, usando os módulos de treinamento validados no ambiente tridimensional de operação de catarata gerado pelo computador Eyesi (VRmagic $\circledast$, Alemanha) para residentes. Realizaram uma pesquisa transversal após o curso para participantes e não participantes com o objetivo de identificar as barreiras percebidas pelos residentes no aprendizado dos procedimentos de facoemulsificação, e se o treinamento de simulação no Eyesi mudou essas percepções.

Os autores identificaram as principais barreiras de aprendizado na cirurgia de extração de catarata por facoemulsificação percebidas pelos residentes oftalmológicos em Hong Kong, era esperado que os residentes mais avançados, e os estagiários que conseguissem completar a facoemulsificação a maior parte do tempo sem precisar de intervenção médica, também tivessem os menores escores de dificuldade nas tarefas cirúrgicas ${ }^{9}$.

No entanto, após o ajuste para esses dois potenciais fatores de confusão, a simulação de treinamento no Eyesi foi significativamente associada ao aumento da confiança em tarefas cirúrgicas de maior dificuldade. $O$ treinamento de simulação em realidade virtual nos módulos validados de Eyesi, seguido de avaliação de proficiência, pareceu ser efetivo na redução das dificuldades percebidas na realização dos procedimentos de facoemulsificação mais exigentes em termos de habilidades em pacientes reais, conforme avaliado pelos residentes ${ }^{9}$.

Os autores ${ }^{9}$ concluem que o objetivo final do uso do simulador é melhorar a segurança do paciente e os resultados por parte dos estagiários/estudantes. Dada a ampla adoção de treinamento baseado em simulador por universidades e centros oftálmicos terciários em muitas partes do mundo, há uma necessidade iminente de um estudo clínico robusto para justificar a eficácia da implementação de módulos de treinamento em simulador de realidade virtual em programas estruturados de treinamento de cirurgia de facoemulsificação.

No estudo de Staropoli et al.[13] a hipótese era que o treinamento de simulação realizado antes da primeira cirurgia de catarata reduziria as taxas de complicações 
durante a rotação inicial de catarata no segundo ano de residência em oftalmologia para residentes do terceiro ano de pós-graduação.

O treinamento no simulador Eyesi tornou-se obrigatório para residentes de oftalmologia do terceiro ano de pós-graduação antes da cirurgia de catarata na instituição dos autores (University of Miami Miller School of Medicine). O estudo consistiu na avaliação das taxas de complicação de 11 residentes treinados em simulador (grupo de estudo) comparadas com seus 11 predecessores imediatos simulados (grupo de comparação). Os dados de complicação foram obtidos dos registros de Morbidade e Mortalidade e comparados usando o teste exato de Fisher e um questionário foi direcionado aos residentes na avaliação de utilidade percebida do treinamento de simulação ${ }^{10}$.

O estudo procurou redução significativa nas complicações da cirurgia de catarata ao vivo, associada à adição de treinamento em simulação cirúrgica. Concluíram que qualquer complicação, PCT e taxas de prolapso vítreo foram menores após o treinamento de simulação $(P=0,037, P=0,032, P=0,032$, respectivamente). As roturas capsulares posteriores e o prolapso vítreo determinam a significância de qualquer análise de complicação e foram relatadas como as complicações mais comuns em cirurgias de catarata realizadas pelos residentes ${ }^{10}$.

Para a pesquisa de Oflaz, Köktekir e Okudan[14] a cirurgia de catarata é um dos procedimentos cirúrgicos mais comuns em oftalmologia, onde o procedimento requer boa coordenação e uma longa curva de aprendizado. Os autores concordam que numerosos estudos indicam que o simulador e o treinamento em laboratório aumentam o desempenho cirúrgico, encurtam a curva de aprendizado dos residentes e reduzem as complicações relacionadas ao procedimento in vivo.

Os autores concluíram que os simuladores podem encontrar um lugar na prática, pois permitem que os treinadores expliquem aspectos da técnica cirúrgica para residentes inexperientes, sem restrições de tempo, e os mesmos podem observar livremente a técnica em questão. Como os pacientes reais não estão envolvidos no procedimento, 
os simuladores fornecem um ambiente menos estressante e mais conveniente tanto para os residentes quanto para os treinadores ${ }^{11}$.

Realizar o procedimento primeiro no simulador e depois em pacientes reais pode ser mais apropriado do ponto de vista ético. Ele instila a autoconfiança no aluno antes de operar em pacientes reais e ajuda a prevenir alguns dos problemas médico-legais em potencial. Em suma, o treinamento em simulador é ideal para os médicos estimularem a confiança do cirurgião antes de procedimentos cirúrgicos reais e prevenir possíveis complicações ${ }^{11}$.

Wisse et al.[15] fizeram um estudo de coorte prospectivo onde a análise comparativa, utilizando um teste t pareado, demonstrou um aumento significativo na eficácia em relação à cirurgia de catarata na realidade após a conclusão do treinamento de catarata no simulador $(p=0,005)$. Além disso, encontraram uma correlação significativa entre o total de tarefas para completar o treinamento de catarata e a autoeficácia pontuada após o trabalho com o simulador $(p=0,038)$. A motivação em relação ao simulador permaneceu estável ao longo do tempo e não pareceu ser influenciada pelo desempenho do simulador ou da vida real.

Foram encontradas evidências de que o desempenho no simulador se correlacionou com a auto eficácia dos residentes, pontuada após o treinamento do simulador, suportando a teoria de que a autoeficácia é determinada pelo desempenho anterior, e esta pareceu, para os autores, inversamente relacionada à facilidade de realização de uma tarefa: a entrega de um esforço maior leva a uma maior satisfação e a uma maior autoeficácia percebida em relação a essa tarefa específica ${ }^{12}$.

Thomsen et al.[16] fez uma pesquisa para delinear a correlação entre a performance da cirurgia de catarata entre um simulador de realidade virtual e uma cirurgia da vida real usando duas ferramentas objetivas de avaliação com evidência de validade.

Cirurgiões de catarata com diferentes níveis de experiência foram incluídos no estudo. Todos os participantes realizaram e gravaram três cirurgias de catarata padrão antes de concluírem um teste de proficiência no simulador de realidade virtual EyeSi. 
Cirurgias de catarata padrão foram definidas como: (1) cirurgia realizada sob anestesia local, (2) idade do paciente> 60 anos e (3) acuidade visual> 1/60 no préoperatório.

Um escore de rastreamento de movimento foi calculado multiplicando-se o comprimento médio do percurso e o número médio de movimentos dos três vídeos cirúrgicos reais de procedimentos completos. O teste EyeSi consistiu em cinco módulos abstratos e dois de procedimento: navegação intracapsular, treinamento antitremor, treinamento antitremor intracapsular, treinamento de fórceps, treinamento bimanual, capsulorrexe e facoemulsificação, dividir e conquistar. Onze cirurgiões foram inscritos. Após um período designado de aquecimento, o teste de proficiência no simulador EyeSi foi fortemente correlacionado ao desempenho em tempo real medido pelo software de rastreamento de movimento de vídeos cirúrgicos de catarata com um coeficiente de correlação de Pearson de -0,70 $(p=0,017)^{13}$.

O desempenho no simulador EyeSi é significativamente e altamente correlacionado ao desempenho cirúrgico real, no entanto, recomenda-se que as avaliações de desempenho sejam feitas usando várias fontes de dados.

No ano seguinte, Thomsen et al.[17] avaliou cirurgiões com níveis de aptidão e experiência cirúrgica variável. A amostra foi de 18 participantes onde foi efetuado o treinamento cirúrgico de catarata em um simulador de realidade virtual (EyeSi) até que um teste baseado em proficiência fosse aprovado. O desfecho primário foi 0 desempenho técnico, medido pela escala de avaliação Objetive Structured Assessment of Cataract Surgical Skill (OSACSS). A escala de classificação consiste em itens específicos da tarefa e índices globais, que são classificados de 1 ponto "desempenho inadequado" a 5 pontos "bem executado". Assim, a avaliação do desempenho técnico incluiu 13 itens específicos de tarefas, que foram classificados usando a escala de classificação de 5 pontos original.

Os resultados deste estudo sugerem que o treinamento em realidade virtual baseado em proficiência pode melhorar o desempenho cirúrgico, não apenas dos cirurgiões iniciantes, mas também dos cirurgiões em um nível intermediário. A conclusão que 
pode ser tirada, é que a melhoria das habilidades técnicas é transferível de uma configuração simulada para a sala de cirurgia ${ }^{14}$.

Segundo o estudo de Chung et al.[18] a aquisição de destreza e o treinamento de habilidades cirúrgicas fora do centro cirúrgico é um tópico crescente no campo da educação dos residentes. Foi demonstrado que o hábito de jogar videogame melhora as habilidades motoras e a coordenação de movimentos em testes de destreza manual. Embora estudos individuais sobre o efeito dos videogames em habilidades de simulação cirúrgica tenham sido controversos, várias revisões sugerem que o ato de jogar semanalmente videogames beneficia o aprendizado do cirurgião, aprimora o tempo cirúrgico e reduz o número de erros em simuladores laparoscópicos.

Desta forma o estudo visou determinar o efeito da atividade motora fina e do treinamento não dominante nas mãos no desempenho do simulador cirúrgico de catarata (Eyesi) em um estudo prospectivo controlado na Universidade de lowa e Veterans Affairs Health Care Systems, lowa City, lowa, EUA. O método consistiu em preenchimento de questionário por estudantes de medicina e de avaliação de destreza microcirúrgica na linha de base usando as três tarefas do simulador cirúrgico: navegação, fórceps e destreza com as duas mãos ${ }^{15}$.

Os participantes foram randomizados no grupo de controle ou intervenção, que consistia em escrever, completar um labirinto, comer e escovar os dentes uma vez por dia com a mão não dominante. Retornaram 4 semanas após a avaliação inicial para o teste de acompanhamento do simulador. Os resultados corroboraram que os jogadores regulares de videogame tiveram escores mais altos do que os não jogadores na navegação $(P=0.021)$ e tarefas bimanuais $(P=0.089)^{15}$.

Todos os participantes apresentaram melhorias estatisticamente significativas em todas as 3 tarefas no acompanhamento após uma única avaliação inicial no simulador cirúrgico (navegação: $P<0,004$; fórceps: $P<0,001$; bimanual: $P<0,004$ ). treinamento com mão não dominante com atividades diárias não mostrou diferenças estatisticamente significantes para mãos dominantes ou mãos não dominantes. 0 grupo de intervenção $(n=17)$ tendeu para uma melhora maior do que o grupo controle 
$(n=16)$ na navegação $(14,78$ versus 7,$06 ; P=0,445)$ e tarefas bimanuais ( 15,2 versus $6,0 ; P=0.324)$ no follow-up (CHUNG et al., 2017).

Os autores concluíram que o jogo de videogame comum melhora o desempenho microcirúrgico de referência medido no simulador cirúrgico. O desempenho da simulação melhorou significativamente no grupo de intervenção e no grupo de controle após uma (01) sessão no simulador. Apesar de não ser estatisticamente significante, treinar a mão não dominante com atividades diárias mostrou uma tendência de melhora na navegação e no desempenho ambidestro ${ }^{15}$.

Landis et al.[19] tiveram o propósito de investigar o impacto do treinamento de residentes com um simulador cirúrgico de catarata sob a percepção dos pacientes sobre o envolvimento do residente na cirurgia de catarata e identificar as características do paciente associadas à disposição de realizar uma cirurgia de catarata realizada por residentes.

Uma pesquisa anônima de 26 perguntas foi distribuída para 430 pacientes consecutivos no Centro Hershey do Estado da Pensilvânia, EUA. A pesquisa incluiu informações demográficas, questões avaliando a disposição de ter um residente envolvido em cirurgia de catarata e questões avaliando o conhecimento do papel dos residentes no atendimento ao paciente. Os pacientes foram distribuídos aleatoriamente para um dos dois grupos. Pacientes designados para o grupo um assistiram a um breve vídeo explicando o papel de um simulador cirúrgico no treinamento de residentes, e foram então solicitados a completar a pesquisa. Os pacientes designados para o grupo dois foram solicitados a concluir a pesquisa sem assistir ao vídeo. O teste t padrão foi usado para comparar dados demográficos. Razões de possibilidades (O.R. [odds ratio]) foram usadas para comparar as respostas entre os dois grupos ${ }^{16}$.

Quatrocentos e dez pacientes (95,3\%) completaram a pesquisa, incluindo 203 pacientes no grupo um e 207 pacientes no grupo dois. Em comparação com os pacientes do grupo dois, os pacientes do grupo um tinham duas vezes mais probabilidade de expressar disposição para um residente em realizar sua cirurgia de 
catarata (O.R. 2,02; $p<0,001)$. Em todos os pacientes, os homens eram mais propensos do que as mulheres a expressar uma disposição para um residente realizar sua cirurgia de catarata (O.R. 1,$65 ; p=0,0065)$. No geral, $25 \%$ dos pacientes expressaram disposição para permitir que um residente realize sua cirurgia de catarata, e esse percentual aumentou para $54 \%$ se os pacientes foram informados que um cirurgião experiente em catarata supervisiona o residente. Noventa e cinco por cento dos pacientes sentiram que deveriam ser informados com antecedência se a cirurgia de catarata era para ser realizada por um residente ${ }^{16}$.

Os pacientes eram mais propensos a expressar disposição em permitir que um residente realizasse sua cirurgia de catarata após assistir a um vídeo explicando o papel de um simulador cirúrgico no treinamento de residentes para cirurgia de catarata. Um processo completo de consentimento informado, incluindo informações sobre a supervisão da cirurgia de catarata realizada por residentes e um breve vídeo detalhando o treinamento dos residentes com um simulador cirúrgico, pode aumentar a disposição do paciente em permitir a participação dos residentes na cirurgia de catarata $^{16}$.

Sikder et al.[20] em sua revisão de literatura mencionam que os simuladores virtuais têm sido amplamente implementados no treinamento médico e cirúrgico, incluindo a oftalmologia. $\mathrm{O}$ crescente número de artigos publicados neste campo requer uma revisão dos resultados disponíveis para avaliar a tecnologia atual e explorar oportunidades futuras.

Conduziram uma pesquisa no PubMed e um total de 10 artigos foram revisados. Os simuladores virtuais mostraram validade de construto em muitos módulos, diferenciando com sucesso os níveis de experiência do usuário durante a cirurgia de facoemulsificação simulada. Os simuladores também mostraram melhorias no desempenho do laboratório de laboratório. A implementação de simuladores no treinamento de residência tem sido associada a uma diminuição nas taxas de complicações da cirurgia de catarata ${ }^{17}$. 
Os autores concluíram que os simuladores de realidade virtual são uma ferramenta eficaz para aferir o desempenho e diferenciar o nível de habilidade dos alunos/residentes. Além disso, eles podem ser úteis na melhora da habilidade cirúrgica e os resultados do paciente na cirurgia de catarata. Oportunidades futuras contam com o aproveitamento de melhorias técnicas em simuladores para educação e pesquisa ${ }^{17}$.

O objetivo do estudo de Lam, Sundaraj e Sulaiman[21] foi revisar a capacidade dos simuladores de realidade virtual na aplicação do treinamento de cirurgia de catarata por facoemulsificação. A revisão incluiu publicações científicas sobre simuladores de cirurgia de catarata que foram desenvolvidas por diferentes grupos de pesquisadores, juntamente com produtos de treinamento cirúrgico comercializados, como EYESI@ e PhacoVision®.

A revisão abrangeu a simulação dos principais procedimentos cirúrgicos de catarata, isto é, incisão corneana, capsulorrexe, fasciculação e implante de lente intraocular em vários simuladores de cirurgia de realidade virtual. Realismo háptico e realismo visual dos procedimentos são os principais elementos para imitar o ambiente cirúrgico real. O envolvimento da oftalmologia na pesquisa em realidade virtual desde o início da década de 1990 tem causado grande impacto no desenvolvimento de simuladores cirúrgicos ${ }^{18}$.

Concluíram que a maioria dos mais recentes sistemas de treinamento de cirurgia de catarata são capazes de oferecer alta fidelidade em feedback visual e retroalimentação háptica, mas o realismo visual, como os movimentos rotacionais de um globo ocular com resposta à força aplicada por instrumentos cirúrgicos, ainda falta em alguns deles. A avaliação das tarefas cirúrgicas realizadas nos simuladores mostrou diferença significativa no desempenho antes e após o treinamento ${ }^{18}$.

\section{CONSIDERAÇÕES FINAIS}

Os programas de treinamento em realidade virtual ( $R V)$ baseados em evidências foram utilizados com sucesso no treinamento de cirurgiões iniciantes em outros 
campos da cirurgia. Alguns estudos oftalmológicos menores estabeleceram validade de construto para um número limitado de tarefas no simulador de RV, comparando principalmente novatos com cirurgiões experientes.

Nosso estudo tornou claro que o treinamento é útil na melhora da acuidade e resultados semelhantes foram relatados tanto para indivíduos mais experientes em oftalmologia, como residentes. O aprendizado rápido também é comum para outras tarefas de simulador cirúrgico. $\mathrm{O}$ treinamento em simulador mostrou-se benéfico no desempenho clínico precoce em outros campos médicos, como colonoscopia e cirurgia laparoscópica.

Seymour et al. (2002) mostraram que danos nos tecidos, como lesões e queimaduras, eram cinco vezes mais prováveis de ocorrer no grupo não treinado em comparação com o grupo treinado em RV. Nos dois módulos estudados, os alunos aprenderam a lidar com o instrumento com mais eficiência e cautela dentro do olho do modelo. $\mathrm{O}$ simulador, portanto, tem o potencial de fazer parte do treinamento inicial de novos cirurgiões de catarata.

A aplicação de simuladores de RV não é uma tecnologia totalmente nova, mas sua aplicação na área de cirurgia de catarata por facoemulsificação para fins de treinamento e avaliação cirúrgicos virtuais ainda está em sua fase inicial. Os resultados dos estudos experimentais apresentados nesta revisão de literatura demonstram que os simuladores de RV têm o potencial e a capacidade de serem aplicados como uma ferramenta viável de avaliação de proficiência nos quatro principais procedimentos completos da cirurgia de catarata por facoemulsificação.

Os autores que compararam os simuladores descobriram, por exemplo, que o desempenho do simulador Eyesi estava significativamente correlacionado com o desempenho cirúrgico de catarata na realidade. Isso sugere que o aumento do uso de simuladores de RV na avaliação da competência cirúrgica pode ser justificado. Além disso, indica que o simulador Eyesi pode ser uma ferramenta valiosa para avaliar a competência cirúrgica. 


\section{REFERÊNCIAS}

BERGQVIST, Joel et al. Establishment of a validated training programme on the $E$ yesi cataract simulator. A prospective randomized study. Acta ophthalmologica, v. 92, n. 7, p. 629-634, 2014.

CHUNG, Anthony T. et al. Effect of fine-motor-skill activities on surgical simulator performance. Journal of Cataract \& Refractive Surgery, v. 43, n. 7, p. 915-922, 2017.

DAWSON, Steven. Perspectives on performance assessment in medical simulation. the surgeon, v. 9, p. S21-S22, 2011.

FEUDNER, Elisabeth M. et al. Virtual reality training improves wet-lab performance of capsulorhexis: results of a randomized, controlled study. Graefe's Archive for Clinical and Experimental Ophthalmology, v. 247, n. 7, p. 955, 2009.

GRANTCHAROV, Teodor P.; FUNCH-JENSEN, Peter. Can everyone achieve proficiency with the laparoscopic technique? Learning curve patterns in technical skills acquisition. The American Journal of Surgery, v. 197, n. 4, p. 447-449, 2009.

HOSLER, Matthew R. et al. Impact of resident participation in cataract surgery on operative time and cost. Ophthalmology, v. 119, n. 1, p. 95-98, 2012.

LAM, Chee Kiang; SUNDARAJ, Kenneth; SULAIMAN, Mohd Nazri. A systematic review of phacoemulsification cataract surgery in virtual reality simulators. Medicina (Kaunas, Lithuania), v. 49, n. 1, p. 1-8, 2013.

LANDIS, Zachary C. et al. Impact of Surgical Simulator Training on Patients' Perceptions of Resident Involvement in Cataract Surgery. Investigative Ophthalmology \& Visual Science, v. 56, n. 7, p. 130-130, 2015.

LE, Tran DB; ADATIA, Feisal A.; LAM, Wai-Ching. Virtual reality ophthalmic surgical simulation as a feasible training and assessment tool: results of a multicentre 
study. Canadian Journal of Ophthalmology/Journal Canadien d'Ophtalmologie, v. 46, n. 1 , p. $56-60,2011$.

LEE, Jiahn-Shing et al. A different approach to assess resident phacoemulsification learning curve: analysis of both completion and complication rates. Eye, v. 23, n. 3, p. 683, 2009.

LIMBURG, Hans; SILVA, Juan C.; FOSTER, Allen. Cataract in Latin America: findings from nine recent surveys. Revista Panamericana de Salud Pública, v. 25, p. 449455, 2009.

NG, Danny Siu-Chun et al. impact of virtual reality simulation on learning barriers of phacoemulsification perceived by residents. Clinical Ophthalmology (Auckland, NZ), v. 12, p. 885, 2018.

OFLAZ, Ayşe Bozkurt; KÖKTEKIR, Bengü Ekinci; OKUDAN, Süleyman. Does Cataract Surgery Simulation Correlate with Real-life Experience? Turkish journal of ophthalmology, v. 48, n. 3, p. 122, 2018.

PAHO. PAN AMERICAN HEALTH ORGANIZATION. Health in the Americas: 2012 edition. Regional outlook and country profiles. Washington: PAHO, 2012. Disponível em: $\quad$ www.paho.org/hq/index.php?option=com_content\&view=article\&id=9774:201453rd-directing-council\&ltemid=40507\&lang=en\#official

POKROY, Russell et al. Impact of simulator training on resident cataract surgery. Graefe's Archive for Clinical and Experimental Ophthalmology, v. 251, n. 3, p. 777-781, 2013.

SACHDEVA, Reecha; TRABOULSI, Elias I. Performance of patients with deficient stereoacuity on the EYESi microsurgical simulator. American journal of ophthalmology, v. 151, n. 3, p. 427-433. e1, 2011.

SADIDEEN, Hazim et al. Surgical experts: born or made?. International Journal of Surgery, v. 11, n. 9, p. 773-778, 2013. 
SALEH, G. M. et al. The development of a virtual reality training programme for ophthalmology: repeatability and reproducibility (part of the International Forum for Ophthalmic Simulation Studies). Eye, v. 27, n. 11, p. 1269, 2013.

SELVANDER, Madeleine; ÅSMAN, Peter. Ready for OR or not? Human reader supplements Eyesi scoring in cataract surgical skills assessment. Clinical ophthalmology (Auckland, NZ), v. 7, p. 1973, 2013.

SEYMOUR, Neal E. et al. Virtual reality training improves operating room performance: results of a randomized, double-blinded study. Annals of surgery, v. 236, n. 4, p. 458, 2002.

SIKDER, Shameema et al. Surgical simulators in cataract surgery training. British Journal of Ophthalmology, v. 98, n. 2, p. 154-158, 2014.

STAROPOLI, Patrick C. et al. Surgical simulation training reduces intraoperative cataract surgery complications among residents. Simulation in Healthcare, v. 13, n. 1, p. 11-15, 2018.

TECLE, Najib et al. Enhancing Microsurgical Skills Through Deliberate Practice. World Neurosurgery. 2018.

THOMSEN, Ann Sofia Skou Skou et al. Investigating inter-procedural transfer of surgical skills using virtual-reality simulation. Investigative Ophthalmology \& Visual Science, v. 57, n. 12, p. 5827-5827, 2016.

THOMSEN, Ann Sofia Skou et al. Operating room performance improves after proficiency-based virtual reality cataract surgery training. Ophthalmology, v. 124, n. 4, p. 524-531, 2017.

WANG, Wei et al. Cataract surgical rate and socioeconomics: a global study. Investigative ophthalmology \& visual science, v. 57 , n. 14 , p. 5872-5881, 2016. 
WHO - WORLD HEALTH ORGANIZATION. Universal eye health: a global action plan 2014-2019. 2013.

WISSE, Robert PL et al. The Eyesi simulator in training ophthalmology residents: results of a pilot study on self-efficacy, motivation and performance. BMJ Simulation and Technology Enhanced Learning, v. 3, n. 3, p. 111-115, 2017.

\section{APÊNDICE - REFERÊNCIAS DE NOTA DE RODAPÉ}

4. KHALIFA, Yousuf $M$. et al. Virtual reality in ophthalmology training. Survey of ophthalmology, v. 51, n. 3, p. 259-273, 2006.

5. WHO - WORLD HEALTH ORGANIZATION. Universal eye health: a global action plan 2014-2019. 2013.

6. PAHO. PAN AMERICAN HEALTH ORGANIZATION. Health in the Americas: 2012 edition. Regional outlook and country profiles. Washington: PAHO, 2012.

7. LIMBURG, Hans; SILVA, Juan C.; FOSTER, Allen. Cataract in Latin America: findings from nine recent surveys. Revista Panamericana de Salud Pública, v. 25, p. 449-455, 2009.

8. WANG, Wei et al. Cataract surgical rate and socioeconomics: a global study. Investigative ophthalmology \& visual science, v. 57, n. 14, p. 5872-5881, 2016.

9. SADIDEEN, Hazim et al. Surgical experts: born or made?. International Journal of Surgery, v. 11, n. 9, p. 773-778, 2013.

10. TECLE, Najib et al. Enhancing Microsurgical Skills Through Deliberate Practice. World Neurosurgery. 2018.

11. BERGQVIST, Joel et al. Establishment of a validated training programme on the $E$ yesi cataract simulator. A prospective randomized study. Acta ophthalmologica, v. 92, n. 7, p. 629-634, 2014. 
12. NG, Danny Siu-Chun et al. impact of virtual reality simulation on learning barriers of phacoemulsification perceived by residents. Clinical Ophthalmology (Auckland, NZ), v. 12, p. $885,2018$.

13. STAROPOLI, Patrick $C$. et al. Surgical simulation training reduces intraoperative cataract surgery complications among residents. Simulation in Healthcare, v. 13, n. 1, p. 11-15, 2018.

14. OFLAZ, Ayşe Bozkurt; KÖKTEKIR, Bengü Ekinci; OKUDAN, Süleyman. Does Cataract Surgery Simulation Correlate with Real-life Experience? Turkish journal of ophthalmology, v. 48, n. 3, p. 122, 2018.

15. WISSE, Robert PL et al. The Eyesi simulator in training ophthalmology residents: results of a pilot study on self-efficacy, motivation and performance. BMJ Simulation and Technology Enhanced Learning, v. 3, n. 3, p. 111-115, 2017.

16. THOMSEN, Ann Sofia Skou Skou et al. Investigating inter-procedural transfer of surgical skills using virtual-reality simulation. Investigative Ophthalmology \& Visual Science, v. 57, n. 12, p. 5827-5827, 2016.

17. THOMSEN, Ann Sofia Skou et al. Operating room performance improves after proficiency-based virtual reality cataract surgery training. Ophthalmology, v. 124, n. 4, p. 524-531, 2017.

18. CHUNG, Anthony T. et al. Effect of fine-motor-skill activities on surgical simulator performance. Journal of Cataract \& Refractive Surgery, v. 43, n. 7, p. 915-922, 2017.

19. LANDIS, Zachary C. et al. Impact of Surgical Simulator Training on Patients' Perceptions of Resident Involvement in Cataract Surgery. Investigative Ophthalmology \& Visual Science, v. 56, n. 7, p. 130-130, 2015.

20. SIKDER, Shameema et al. Surgical simulators in cataract surgery training. British Journal of Ophthalmology, v. 98, n. 2, p. 154-158, 2014. 
21. LAM, Chee Kiang; SUNDARAJ, Kenneth; SULAIMAN, Mohd Nazri. A systematic review of phacoemulsification cataract surgery in virtual reality simulators. Medicina (Kaunas, Lithuania), v. 49, n. 1, p. 1-8, 2013.

Enviado: Janeiro, 2020.

Aprovado: Janeiro, 2020. 